\title{
Elastic Wave Propagation in Layered Anisotropic Media'
}

\author{
DUN I. ANDERSON \\ Scismoloyical Lathoratory, California Institute of 'T'cchnolog!! \\ Pasadena, California
}

\begin{abstract}
This is an analysis of the dispersive properties of transversely isotropic media. This kind of anisotropy is exhibited by hexagonal crystals, sediments, planar igneous bodies, ice sheets, and rolled metal sheets where the unique axis is perpendicular to the direction of surface wave propagation and the other axes are distributed randomly in the plane of the layers. Period equations are derived for waves of Rayleigh, Stoneley, and Love types, and comparisons are made, in certain cases, with ray theoretical and plane stress solutions. Anisotropy can have a pronounced effect on both the range of existence and the shape of the dispersion curves and can lead to an apparent discrepancy between Love and Rayleigh wave data. Attention is focused in this initial paper on a single solid layer in vacuo (i.e. a free plate) and a solid layer in contact with a fluid halfspace. The single layer solutions are gencralized to $n$-layer media by the use of Haskell matrices.
\end{abstract}

1. Introduction. Elistic wave problems are usually formulated for convenicnce under the restrictive assumptions of homogencity, perfect elasticity, plane parallel boundaries, and isotropy. Although these assumptions are often approximately satisfied in practice, certain ambiguities exist between theory and observation that indicate the need for reformulating some of these problems under less restrictive and possibly more realistic assumptions. Herein we drop the assumption of isotropy.

Anisotropy is exhibited in its purest form in single crystals but also occurs in collections of crystals or minerals which have erystallized or have been deposited with a preferred orientation, or have been subjected to nonuniform forces after formation. A layered medium by its very nature is anisotropic in the large, but the individual layers may also be anisotropic in a manner which cannot be handled by a further subdivision into finer layers. Heterogeneous media with random grain orientation tend to be isotropic.

We shall consider materials that possess an axis of symmetry in the sense that all rays at right angles to this axis are equivalent. Such media are called 'transversely isotropic,' and wave propagation in an infinite or semi-infinite

${ }^{1}$ Contribution 1036, Division of Ceological Sciences, California Institute of Technology, Pasadena, California. medium with this symmetry has been discussed by Love [1944], Satô [1950], Musgrave [1959], and Stoneley [1949]. A convenient summary is given by Mason [1958] and Ewing, Jardetzky, and Press [1957]. Transverse isotropy results in the same set of elastic constants as that for hexagonal symmetry and hence is exhibited in all metals or minerals crystallizing in the hexagonal system. This symmetry is also expected to be displayed by sediments, planar igneous bodies, floating ice sheets, and rolled or extruded motal and plastic sheets. This latter material is often used in two-dimensional model experiments.

An isotropic solid is governed by two elastic constants and the characteristic equation has three ronts, one corresponding to a compressional wave and a double root corresponding to a distortional wave. These velocities are independent of direction. A transversely isotropic solid is governed by five elastic constants, and the separation into two waves, one for which the curl of the displacement vanishes and one for which the divergence of the displacement vanishes, does not in general occur. Corresponding to any assigned wave normal there are three velocities of elastic wave propagation, and only in special cases do these degenerate to purely shear and purely compressional motion.

2. Generalized Hooke's law and the equations of motion. The matrix of the elastic constants for a medium with hexagonal or transverse 
isotropic symmetry is [Love, 19.44]

$\begin{array}{cccccc}c_{11} & c_{12} & c_{13} & 0 & 0 & 0 \\ c_{12} & c_{11} & c_{13} & 0 & 0 & 0 \\ c_{13} & c_{13} & c_{33} & 0 & 0 & 0 \\ 0 & 0 & 0 & c_{14} & 0 & 0 \\ 0 & 0 & 0 & 0 & c_{14} & 0 \\ 0 & 0 & 0 & 0 & 0 \frac{c_{11}-c_{12}}{2}\end{array}$

l'or an isotropic body,

$$
\begin{gathered}
c_{11}=c_{33}=\lambda+2 \mu ; \quad c_{13}=\lambda ; \\
\left(c_{11}-c_{12}\right) / 2=c_{14}=\mu
\end{gathered}
$$

If we ignore body forces, the equations of small motion are three of the form,

$$
\rho \frac{\partial^{2} u}{\partial t^{2}}=\frac{\partial p_{x x}}{\partial x}+\frac{\partial p_{x y}}{\partial y}+\frac{\partial p_{x z}}{\partial z}
$$

The stresses $p_{i j}$ are derived from the strain encrgy function $W$ by

$$
p_{x x}=\frac{\partial W}{\partial e_{x x}}, \quad p_{x y}=\frac{\partial W}{\partial e_{x y}}, \text { etc. }
$$

where [Love, 1944]

$$
\begin{aligned}
& 2 W=c_{11}\left(e_{x x}^{2}+e_{y y}^{2}\right)+c_{33} e_{z z}^{2} \\
& \quad+2 c_{13}\left(e_{x x}+e_{y y}\right) e_{z z}+2 c_{12} e_{x x} e_{y y} \\
& \quad+c_{44}\left(e_{y z}^{2}+e_{z x}^{2}\right)+\frac{1}{2}\left(c_{11}-c_{12}\right) e^{2}{ }_{j y}
\end{aligned}
$$

The stresses are, accordingly,

obvious that $z$ has been taken as the unique axis.

The equations of motion become

$$
\begin{aligned}
\rho \frac{\partial^{2} u}{\partial t^{2}} & =\frac{\partial}{\partial x}\left(c_{11} \frac{\partial u}{\partial x}+c_{12} \frac{\partial v}{\partial y}+c_{13} \frac{\partial w}{\partial z}\right) \\
& +\frac{\partial}{\partial y}\left(\frac{c_{11}-c_{12}}{2}\right) \frac{\partial u}{\partial y}+\frac{\partial}{\partial z} c_{44} \frac{\partial w}{\partial x} \\
\rho \frac{\partial^{2} v}{\partial t^{2}}= & \left(\frac{c_{11}-c_{12}}{2}\right) \frac{\partial^{2} w}{\partial x \partial y}+\frac{\partial}{\partial y}\left\{c_{12} \frac{\partial^{2} u}{\partial x^{2}}\right. \\
& \left.+c_{11} \frac{\partial^{2} v}{\partial y^{2}}+c_{13} \frac{\partial^{2} w}{\partial z^{2}}\right\}+c_{14} \frac{\partial^{2} v}{\partial z^{2}} \\
\rho \frac{\partial^{2} w}{\partial t^{2}} & =c_{44} \frac{\partial^{2} w}{\partial x^{2}}+c_{44} \frac{\partial^{2} v}{\partial y \partial z} \\
+ & \frac{\partial}{\partial z}\left(c_{13} \frac{\partial^{2} u}{\partial x^{2}}+c_{13} \frac{\partial^{2} v}{\partial y^{2}}+c_{33} \frac{\partial^{2} w}{\partial z^{2}}\right)
\end{aligned}
$$

where $u, v, w$ are the displacements in the $x, y, z$ directions. There is no advantage in introducing the standard potentials since the equations of motion are still unseparable.

3. Plane waves in an infinite medium. The theory of plane wave propagation in the interior of an infinite anisotropic body is well developed (see, for instance, Love [1944] or Mason [1958]). We begin with a brief review of this theory to cstablish the setting for the following sections.

For plane waves propagated in a direction specified by direction cosines $(l, m, n)$ we take

$$
(u, v, w)=(U, V, W) e^{i \omega t} e^{-i k(t x+m y+n s)}
$$

Substitution into the equations of motion gives

$$
\left[\begin{array}{ccc}
\mathcal{F}-\rho c^{2} & \operatorname{lm}\left(\frac{c_{11}+c_{12}}{2}\right) & n l\left(c_{13}+c_{14}\right) \\
\operatorname{lm}\left(\frac{c_{11}+c_{12}}{2}\right) & S-\rho c^{2} & m n\left(c_{13}+c_{44}\right) \\
n l\left(c_{13}+c_{44}\right) & m n\left(c_{13}+c_{44}\right) & 3 C-\rho c^{2}
\end{array}\right]\left[\begin{array}{c}
U \\
V \\
W
\end{array}\right]=0
$$

$$
\begin{aligned}
& p_{x x}=c_{11} e_{x x}+c_{12} e_{y y}+c_{13} e_{z z} \\
& p_{y y}=c_{12} e_{x x}+c_{11} e_{y y}+c_{13} e_{z z} \\
& p_{z z}=c_{13}\left(e_{x x}+e_{y y}\right)+c_{33} e_{x z} \\
& p_{y x}=p_{x y}=\frac{1}{2}\left(c_{11}-c_{12}\right) e_{x y} \\
& p_{z y}=p_{y z}=c_{44} e_{y z} \\
& p_{x z}=p_{z x}=c_{44} e_{y z}
\end{aligned}
$$

From the symmetry of the above equations it is where

$$
\begin{aligned}
\mathcal{F} & =l^{2} c_{11}+m^{2}\left(\frac{c_{11}-c_{12}}{2}\right)+n^{2} c_{44} . \\
\mathcal{S} & =l^{2}\left(\frac{c_{11}-c_{12}}{2}\right)+m^{2} c_{11}+n^{2} c_{44} . \\
\mathcal{H} & =\left(l^{2}+m^{2}\right) c_{44}+n^{2} c_{33} .
\end{aligned}
$$

By setting the determinant of the coefficients 
equal to zero, we obtain the velocity equation. Two special cases may be dealt with immediately:

(a) For transmission along the unique axis, $n=1, m=l=0, c^{2}=c_{33} / \rho$, and $c^{2}=c_{44} / \rho$ are solutions. The first corresponds to a vertically traveling purely compressional wave $(P V)$ and the second is a double root corresponding to a vertically traveling shear wave with horizontal particle motion. The degeneracy is caused by the $S V$ and $S H$ waves becoming indistinguishable.

(b) For transmission along the $x$ or $y$ direction or any other direction perpendicular to the $\boldsymbol{z}$ axis, $n=0$, the solutions are

$$
\begin{array}{rlrl}
c^{2} & =\frac{c_{11}}{\rho} & \text { compressional, } & P I I \\
c^{2} & =\frac{c_{14}}{\rho} & \text { shear, } S V \\
c^{2} & =\frac{c_{11}-c_{12}}{2 \rho} & \text { shear, } S I I
\end{array}
$$

Therefore, measurement along these two directions will determine four of the five elastic constants. To determine the fifth we need a measurement at some intermediate angle. In particular we can set $l=n=1 / \sqrt{2}, m=0$, and from the velocity equation obtain

$$
\begin{aligned}
c_{13}=\left\{\left[2 \rho c^{2}-\frac{1}{2}\left(c_{11}\right.\right.\right. & \left.\left.+c_{33}+2 c_{44}\right)\right]^{2} \\
& \left.-\frac{1}{2}\left(c_{11}-c_{33}\right)^{2}\right\}^{1 / 2}-c_{44}
\end{aligned}
$$

giving $c_{18}$ in terms of the velocity of the fastest wave traveling at $45^{\circ}$ to the $z$ axis. Solving the velocity equation for arbitrary $l, m, n$, we can determine the directional dependence of the wave velocities.

In the following we shall use the designations

$$
\begin{gathered}
c_{33} / \rho=\alpha_{2}{ }^{2} \quad(l V) ; \quad c_{11} / \rho=\alpha_{1}{ }^{2} \\
c_{44} / \rho=\beta_{1}{ }^{2} \quad\left(S I_{V}, S V_{V}, S V_{H I}\right) ; \\
\left(c_{11}-c_{12}\right) / 2 \rho=\beta_{2}{ }^{2} \quad\left(S H_{H}\right)
\end{gathered}
$$

For an isotropic body

$$
\alpha_{1}^{2}=\alpha_{2}^{2}=(\lambda+2 \mu) / \rho
$$

and

$$
{\beta_{1}}^{2}={\beta_{2}}^{2}=\mu / \rho
$$

4. Surface waves in an anisotropic layer. Consiller now a layer of thickness $2 H$ with the alsove symmetry overlying a fluid halfspace with constants $\rho_{2}, \lambda_{2}$. Take $z$ increasing downward from the center of the layer. This configuration will permit us to study the effect of anisotropy in a relatively simple system for which the isotropic theory is well developed and for which experimental data are available. Also, with this general case in hand we can easily study as special cases the effect of anisotropy on Rayleigh and Stoneley waves and on propagation in a free plate. Later we shall discuss the general $n$-layer anisotropic problem and point out how anisotropy will introduce apparent discrepancies between Love and Rayleigh wave data as well as giving erroneous results for Love or Rayleigh data used alone. Since we shall be interested in applying the results of our present restricted problem to a high-speed layer overlying a lowspeed fluid halfspace (the floating ice sheet problem) we have the additional problem of leakage for all modes with phase velocities greater than the fluid velocity, but this is resolved by programming our resultant period equation in complex algebra, thus permitting the location of complex roots. This, however, introduces no additional difficulties into our present analysis.

Restricting ourselves to motion in two dimensions $(x, z)$ we put $\partial / \partial y=0, e_{\nu y}=0, e_{\nu z}=0$.

For surface waves we seek solutions of the type

$$
(u, w)=\left[U_{i}(z), W_{i}(z)\right] e^{i(\omega t-k x)}
$$

Substitution into the equations of motion yields

$$
\begin{aligned}
& -\rho_{1} \omega^{2} U(z)=-c_{11} k^{2} U(z) \\
& -i k\left(c_{13}+c_{44}\right) W^{\prime}(z)+c_{44} U^{\prime \prime}(z) \\
& -\rho_{1} \omega^{2} W(z)=c_{33} W^{\prime \prime}(z) \\
& -i k\left(c_{13}+c_{44}\right) U^{\prime}(z)-k^{2}{ }^{2}{ }^{\prime} 14
\end{aligned}
$$

where the primes denote $\partial / \partial z$.

If $U(z)=U e^{r_{i}}$ and $W(z)=i W_{e^{\prime}{ }^{z}}$, then

$$
\begin{aligned}
& -\rho_{1} \omega^{2} U=-c_{11} U+k \nu_{i} G W+c_{41} \nu_{i}^{2} U \\
& -\rho_{1} \omega^{2} W=c_{33} \nu_{i}{ }^{2} W-k \nu_{i} G U-k^{2} c_{44} W
\end{aligned}
$$

where $G=c_{13}+c_{14}$.

The condition that nonzero roots of equation 10 exist is

$$
\begin{array}{r}
\left(c_{41} \nu_{i}^{2}-c_{11} k^{2}+\rho_{1} \omega^{2}\right)\left(c_{33} \nu_{i}{ }^{2}-k^{2} c_{44}+\rho_{1} \omega^{2}\right) \\
+k^{2} \nu_{i}^{2} l^{2}
\end{array}
$$


The explicit values of $\nu^{2}$ are

where

$$
\nu_{i}^{2}=-\frac{M_{1}}{2 c_{33} c_{44}} \pm \frac{M_{3}}{2 c_{33} c_{44}}
$$

$$
\begin{aligned}
& M_{3}^{2}=M_{1}{ }^{2}-4 M_{2} c_{33} c_{44} . \\
& M_{1}=c_{33}\left(\rho_{1} \omega^{2}-c_{11} k^{2}\right) \\
& \quad+c_{44}\left(\rho_{1} \omega^{2}-c_{44} k^{2}\right)+k^{2} G^{2} . \\
& M_{2}=\left(\rho_{1} \omega^{2}-c_{11} k^{2}\right)\left(\rho_{1} \omega^{2}-c_{44} k^{2}\right) .
\end{aligned}
$$

For an isotropic body equation 12 becomes

$$
\begin{array}{r}
\nu_{1}{ }^{2}=\left(k^{2}-\omega^{2} / \alpha^{2}\right), \quad \nu_{2}{ }^{2}=\left(k^{2}-\omega^{2} / \beta^{2}\right), \\
\alpha^{2}=(\lambda+2 \mu) / \rho, \quad \beta^{2}=\mu / \rho
\end{array}
$$

so that $\nu_{1}$ reduces to the form associated with a pure compressional wave and $\nu_{2}$ reduces to that for a pure distortional wave.

For a given $\nu_{i}$ the displacement ratios in the in the solid, and

$$
\begin{aligned}
U^{*} & =U_{0} e^{-\nu^{\prime} z} \\
W^{*} & =-\left(i \nu^{\prime} / k\right) U_{0} e^{-\nu^{\prime} z}
\end{aligned}
$$

in the liquid, where $\nu^{\prime 2}=\left(k^{2}-\omega^{2} / \alpha^{*_{2}}\right)$ and $\alpha^{*_{2}}=\lambda_{2} / \rho_{2}$.

The boundary conditions are

$p_{::}=c_{33} \frac{\partial W}{\partial z}+c_{13} \frac{\partial U}{\partial x}=0 ; \quad z=-H$

$p_{z x}=c_{t 4}\left(\frac{\partial U}{\partial z}+\frac{\partial W}{\partial x}\right)=0 ; \quad z=-H$

$p_{z x}=0 ; \quad p_{z z}=p^{*}{ }_{2 z} ; \quad W=W^{*} ; \quad z=H$

Substituting equations 14 into equations 15 gives

$$
\begin{aligned}
& {\left[I_{1}\left[-\gamma_{1} \nu_{1} c_{33}+c_{13} h\right] s h \nu_{1} I I+U_{2}\left[\gamma_{1} \nu_{1} c_{33}-c_{13} k\right] c h \nu_{1} I I+U_{33}\left[-\nu_{2} \gamma_{2} c_{33}+c_{13} k\right] s h \nu_{2} l l\right.} \\
& +U_{4}\left[\nu_{2} \gamma_{2:} c_{33}-c_{13} k\right]_{c}: h \nu_{2} I I=0 \\
& U_{1}\left[\nu_{1}+k \gamma_{1}\right] c h \nu_{1} I I+U_{2}\left[-\nu_{1}-k \gamma_{1}\right] s h \nu_{1} I I+U_{3}\left[\nu_{22}+\gamma_{2} k\right] c h \nu_{2} I I+U_{4}\left[-\nu_{2}-\gamma_{2} h\right] s h \nu_{2} I I=0 \\
& U_{1}\left[\gamma_{1} \nu_{1} c_{33}-c_{13} k\right] \operatorname{sh} \nu_{1} H+U_{2}\left[c_{33} \gamma_{1} \nu_{1}-c_{13} k\right] \operatorname{ch} \nu_{1} H+U_{3}\left[\nu_{2} c_{33} \gamma_{2}-c_{13} k\right] s h \nu_{2} I I \\
& +U_{4}\left[c_{33} \nu_{2} \gamma_{2}-c_{13} k\right] c h \nu_{2} I I+U_{0} \frac{\lambda_{2}}{k}\left[k^{2}-\nu^{\prime 2}\right] e^{-\nu^{\prime} I I}=0
\end{aligned}
$$

solid are

$$
\begin{gathered}
\left(\begin{array}{l}
U \\
W
\end{array}\right)_{i}=-\frac{k \nu_{i} G}{\left(c_{4+} \nu_{i}^{2}-c_{11} k^{2}+\rho_{1} \omega^{2}\right)} \\
=\frac{\left(c_{33} \nu_{i}^{2}-k^{2} c_{44}+\rho_{1} \omega^{2}\right)}{k \nu_{i} C^{2}}=\frac{1}{\gamma_{i}} \\
W_{i}=\gamma_{i} U_{i}
\end{gathered}
$$

For isotropic media $\gamma_{1}=\nu_{1} / k, \gamma_{2}=k: / \nu_{2}$. We therefore take as our solutions

$$
\begin{aligned}
& U=U_{1} \operatorname{sh} \nu_{1} z+U_{2} \operatorname{ch} \nu_{1} z \\
& +U_{3} \operatorname{sh} \nu_{2} z+U_{4} \operatorname{ch} \nu_{2} z \\
& W=i \gamma_{1} U_{1} \operatorname{ch} \nu_{1} z+i \gamma_{1} U_{2} s h \nu_{1} z \\
& +i \gamma_{2} U_{3} c h \nu_{2} z+i \gamma_{2} U_{4} s h \nu_{2} z
\end{aligned}
$$

The condition that the determinant of the coefficients vanish is the period equation

$$
\begin{aligned}
& \mathrm{II}_{1} \Omega_{2} s h \nu_{1}\left(\mathrm{II}, \Gamma_{2} s h \nu_{2} c h \nu_{1}-\Pi_{2} \Gamma_{1} c h \nu \nu_{2} s h \nu_{1}\right) \\
& +\Lambda_{2} I I_{2} s h \nu_{2}\left(\Pi_{22} \Gamma_{1} s h \nu_{1} c h \nu_{2}\right. \\
& \text { - } \left.\mathrm{II}_{1} \Gamma_{2} s h \nu_{2} \operatorname{ch} \nu_{1}\right) \\
& +\mathrm{II}_{1} \Omega_{1} \operatorname{ch} \nu_{1}\left(\Pi_{1} \Gamma_{{ }_{2}} \operatorname{ch} \nu_{2} s h \nu_{1}\right. \\
& \left.-\mathrm{II}_{2} \Gamma_{1} \operatorname{sh} \nu_{2} \operatorname{ch} \nu_{1}\right) \\
& +\Lambda_{1} \Pi_{2} \operatorname{ch} \nu_{2}\left(\Pi_{2} \Gamma_{1} \operatorname{sh} \nu_{2} c h \nu_{1}\right. \\
& \left.-\Pi_{1} \Gamma_{2} s h \nu_{1} \operatorname{ch} \nu_{2}\right)=0
\end{aligned}
$$

where

$$
\begin{array}{ll}
\Gamma_{1}=\left[-\gamma_{1} \nu_{1} c_{33}+c_{13} k\right] ; \quad \mathrm{II}_{1}=\left[\nu_{1}+k \gamma_{1}\right] \\
\Gamma_{2}=\left[-\nu_{2} \gamma_{2} c_{33}+c_{13} k\right] ; \quad I_{2}=\left[\nu_{2}+k \gamma_{2}\right]
\end{array}
$$




$$
\begin{aligned}
& \Lambda_{1}=\nu^{\prime} \Gamma_{1} \operatorname{sh} \nu_{1}+\lambda_{2} \gamma_{1}\left(k^{2}-\nu_{1}{ }^{2}\right) \operatorname{ch} \nu_{1} \\
& \Lambda_{2}=\nu^{\prime} \Gamma_{1} \operatorname{ch} \nu_{1}+\lambda_{2} \gamma_{1}\left(k^{2}-\nu_{1}{ }^{\prime 2}\right) s h \nu_{1} \\
& \Omega_{1}=\nu^{\prime} \Gamma_{2} \operatorname{sh} \nu_{2}+\lambda_{2} \gamma_{2}\left(k^{2}-\nu_{1}{ }^{\prime 2}\right) \operatorname{ch} \nu_{2} \\
& \Omega_{2}=\nu^{\prime} \Gamma_{2} \operatorname{ch} \nu_{2}+\lambda_{2} \gamma_{2}\left(k^{2}-\nu_{1}{ }^{\prime 2}\right) \operatorname{sh} \nu_{2}
\end{aligned}
$$

For an isotropic layer this yields the period equation given by Press and Ewing [1951].

The asymptotic form of equation 17 for short wavelengths is

$$
\begin{aligned}
& \left(\Pi_{1} \Gamma_{2}-\Pi_{2} \Gamma_{1}\right)\left[\nu^{\prime}\left(\Pi_{1} \Gamma_{2}-\Pi_{2} \Gamma_{1}\right)\right. \\
& \left.\quad+\lambda_{2}\left(k^{2}-\nu_{1}{ }^{\prime 2}\right)\left(\Pi_{1} \gamma_{2}-\Pi_{2} \gamma_{1}\right)\right]=0
\end{aligned}
$$

It will later be shown that the first factor is the Rayleigh equation for anisotropic media and the second factor is the Stoneley equation for the interface between an anisotropic solid and a fluid.

5. Free plate. If $\rho_{2}$ is set equal to zero, the factors of the period equation are

$$
\begin{aligned}
& \left(\frac{\tanh \nu_{1} H}{\tanh \nu_{2} H}\right)^{-1} \\
& \quad=\frac{\left[\nu_{1}+k \gamma_{1}\right]\left[\nu_{2} \gamma_{2} c_{33}-c_{13} k\right]}{\left[\gamma_{1} \nu_{1} c_{33}-c_{13} k\right]\left[\nu_{2}+\gamma_{2} k\right]}=\frac{\Gamma_{2} \Pi_{1}}{\Gamma_{1} \Pi_{2}}
\end{aligned}
$$

the upper sign corresponding to antisymmetrical waves and the lower sign corresponding to symmetrical waves in a free plate.

In the long wavelength limit this becomes

$$
\left[\nu_{1} / \nu_{2}\right]^{ \pm 1}=\Gamma_{2} \Pi_{1} / \Gamma_{1} \Pi_{2}
$$

For symmetrical waves this gives

$$
c_{33}\left(\rho \omega^{2} / k^{2}\right)-\left(c_{11} c_{33}-c_{13}^{2}\right)=0
$$

We can also derive the long wavelength limit for symmetrical waves from plane stress theory. Taking as before the $x y$ plane parallel to the surface of the plane and propagation in the $x$ direction, we have

$$
\partial p_{x x} / \partial x=\rho \partial^{2} u / \partial t^{2}
$$

Eliminating $\partial w / \partial z$ from the first and third of equations 3 yields

$$
p_{x x}=\frac{c_{11} c_{33}-c^{2}{ }_{13}}{c_{33}} \frac{\partial u}{\partial x}
$$

Therefore

$$
\rho \frac{\partial^{2} u}{\partial t^{2}}=\frac{c_{11} c_{33}-c^{2}{ }_{13}}{c_{33}} \frac{\partial^{2} u}{\partial x^{2}}
$$

and the plate velocity $c_{p}$ is given by

$$
c_{p}{ }^{2}=\left(c_{11} c_{33}-c_{13}^{2}\right) / \rho c_{33}
$$

in agreement with $(20 a)$.

This derivation will serve as a check on the low-frequency limit of our ensuing calculations. Note that the plate velocity does not depend only on the horizontal compressional and $S V$ velocities, as we may have suspected for the long wave limit, but involves the constants in the vertical and intermediate directions as well. This can lead to plate velocities which are outside the range possible for an isotropic solid, a fact which holds true also for the rest of the dispersion curve. This is related to the fact that directional Poisson's ratios in an anisotropic solid can exceed 0.5 .

For waves short compared with the layer thickness, the hyperbolic tangents can be replaced by unity, giving

$$
\Gamma_{2} \Pi_{1}-\Gamma_{1} \Pi_{2}=0
$$

for both symmetrical and antisymmetrical waves.

This is, again, the Rayleigh equation for anisotropic media. A more detailed discussion of the free anisotropic plate is in preparation (I. Abubakar, personal communication).

6. Rayleigh and Stoneley waves. Consider an anisotropic halfspace with the above properties. Assume solutions that decrease exponentially with depth ( $z$ is positive downward and the solid lies in the top halfspace):

$$
\begin{aligned}
U & =U_{1} e^{\nu_{1} z}+U_{3} e^{\nu_{2} z} \\
W & =i \gamma_{1} U_{1} e^{\nu_{1} z}+i \gamma_{2} U_{3} e^{\nu_{2} z}
\end{aligned}
$$

The $\nu_{i}$ and $\gamma_{i}$ are the same as were derived previously for the general case. Applying the condition of a traction free surface we obtain the period equation

$$
R=-\Gamma_{1} \Pi_{2}+\Gamma_{2} \Pi_{1}=0
$$

This is the Rayleigh equation for transversely isotropic media first derived by Stoneley [1949]. It reduces in the case of isotropy to

$$
R=\left[\nu_{2}{ }^{2}+k^{2}\right]^{2}-4 \nu_{1} \nu_{2} k^{2}=0
$$

For Stoneley waves we assume motion dying off into both the fluid and the solid. Assume equation 26 for motion in the solid, and, for the fluid.

$$
\begin{aligned}
U^{*} & =U_{0} e^{-\nu^{\prime} z} \\
W^{*} & =-\left(i \nu^{\prime} / k\right) U_{0} e^{-\nu^{\prime} z}
\end{aligned}
$$


Applying the conditions of a stress free interface and continuity of vertical displacement, we obtain the Stoneley period equation ${ }_{y}$

$$
\frac{\lambda_{2}\left(k^{2}-\nu^{\prime 2}\right)}{\nu^{\prime}}=\frac{\Pi_{1} \Gamma_{2}-\mathrm{II}_{2} \Gamma_{1}}{\gamma_{1} \mathrm{II}_{2}-\gamma_{2} \Pi_{1}}
$$

For isotropy this goes into

$$
R+\delta=0
$$

where

$$
\delta=\frac{\rho_{2} \alpha^{*^{2}} \nu_{1}}{\rho_{1} \beta_{1}^{2} \nu^{\prime}}\left(\nu_{2}{ }^{2}-k^{2}\right)\left(\nu^{\prime 2}-k^{2}\right)
$$

This is the form of the Stoneley equation given by Press and Ewing [1951]. When $\rho_{2}=0$, we obtain the Rayleigh equation. Thus, as we indicated earlier, the high-frequency limit of the fundamental symmetric and antisymmetric modes of a free plate is the Rayleigh velocity. A floating plate has two branches of the fundamental mode, one corresponding to the Rayleigh velocity and the other to the Stoneley velocity.

7. Love waves. In a layered transversely isotropic medium Love waves exist independently of Rayleigh waves; that is, there is no coupling between motions of Rayleigh and Love types for waves propagating in a plane perpendicular to the unique axis. This is the lowest symmetry for which this coupling does not, in general, exist.

Considering a free layer and taking the axes as before, we see that the equations of motion can be satisfied by putting $u=w=0, \partial / \partial y=0$. For waves of the Love type we take

$$
\begin{aligned}
& v=V(z) e^{i(\omega t-k: x)} \\
& \left.=\left[v_{1} \operatorname{sh} \zeta z+v_{2} c h \zeta z\right)\right] e^{i(\omega t-k \cdot)}
\end{aligned}
$$

and obtatin the reduced watve equation

$$
\partial^{2} v / \partial t^{2}=\left(N-\zeta c^{2}\right) v k^{2} / L
$$

so that

$$
\zeta^{2}=\left(N-\rho c^{2}\right) k^{2} / L
$$

where $N=\left(c_{11}-c_{12}\right) / 2, L=c_{44}$. When $L_{L}=N=\mu=\rho \beta^{2}$, we have isotroly and

$$
\zeta^{2}=\left(k^{2}-\omega^{2} / \beta^{2}\right)
$$

'The boundary conditions are

$$
\rho_{z u}=L \partial v / \partial z=0 \quad \text { at } \quad z= \pm I I
$$

These conditions loul to the periol expation

$$
\text { tanh } 2 k H\left[\left(N-\rho c^{2}\right) / L\right]^{1 / 2}=0
$$

If $\rho c^{2}<N$, no roots of the above equation exist. Therefore we must have $\rho c^{2} \geq N$, or $c^{2} \geq \beta_{2}^{2}$. The period equation can therefore be written

$$
\tan 2 k H(N / L)^{1 / 2}\left[\left(c^{2} / \beta_{2}{ }^{2}\right)-1\right]^{1 / 2}=0
$$

Equation 36 is satisfied if

$$
\begin{aligned}
& 2 k H(N / L)^{1 / 2}\left[\left(c^{2} / \beta_{2}{ }^{2}\right)-1\right]^{1 / 2} \\
& \quad=n \pi \quad \text { where } n=0,1,2 \ldots
\end{aligned}
$$

which is the Love period equation for a free anisotropic plate.

This differs by a factor of $(N / L)^{1 / 2}$ from the isotropic Love wave period equation. Note that $c=\beta_{2}$ is always a solution. The above derivation also holds for an anisotropic layer in contact with a fluid layer on one or both sides.

As Stoneley [1949] has pointed out, it is the modulus $c_{44}$ that resembles the isotropic rigidity for Rayleigh wave motion, whereas the corresponding modulus for Love wave motion is $\left(c_{11}-c_{12}\right) / 2$. This holds true for layered anisotropic media in general and will make the velocity structure as determined for Rayleigh wave data inconsistent with Love wave information unless the anisotropy is taken into account.

For an anisotropic layer of thickness $2 H$ with constants $L_{1}, N_{1}$, and $\beta_{2}$ over an anisotropic halfspace with constants $L_{2}, N_{2}$, and $\beta^{*}$, we obtain for Love waves

$$
\begin{aligned}
\operatorname{tin} 2 k J I\left(\frac{c^{2}}{\beta_{2}}-1\right)^{1 / 2}\left[\frac{N_{1}}{L_{1}}\right]^{1 / 2} \\
=\left[\frac{L_{12} N_{2}}{L_{1} N_{1}}\right]^{1 / 2}\left[\frac{1-\left(c^{2} / \beta^{* 2}\right)}{c^{2} / \beta_{2}^{2}-1}\right]^{1 / 2}
\end{aligned}
$$

In the corresponding isotropic case $N=L=\mu$, and we recover the Love equation in familiar form. Stoneley's [1949] derivation of the anisotropic Love equation is apparently in error.

It can be shown that the period equation expresses the condition of constructive interference between multireflected plane $S H$ waves. This condition may be written, for the free plate,

$$
4 H \cos \theta=n l_{0}
$$

where $\theta$ is the angle the ray makes with the vertical and $l_{0}$ is the wavelength measured along the ray. The velocity of sll waves satisfies the 
equation

$$
\rho \beta^{2}(\theta)=l^{2} N+n^{2} L
$$

With the substitutions $\beta(\theta) / c=\sin \theta$, $k=2 \pi \sin \theta / l_{0}, \beta_{1}{ }^{2}=L / \rho$, and $\beta_{2}{ }^{2}=N / \rho$ we can write for equations 38 and 39

$$
\begin{gathered}
n \pi=\frac{2 k H c}{\beta(\theta)}\left[1-\frac{\beta^{2}(\theta)}{c^{2}}\right]^{1 / 2} \\
\beta^{2}(\theta)=\frac{L}{N} c^{2}\left(\frac{c^{2}}{\beta_{2}^{2}}-1+\frac{L}{N}\right)^{-1}
\end{gathered}
$$

Substitution of (39a) into (38a) gives the period equation

$$
2 k H(N / L)\left[\left(c^{2} / \beta_{2}{ }^{2}\right)-1\right]^{1 / 2}=n \pi
$$

8. Numerical results. The general period equation (17) was programmed for the Cal Tech Computing Center's Burroughs 220 electronic digital computer, using a complex root-finding subroutine developed by Phinney [1961]. Modifcations were built into the program, making it possible to solve for the symmetrical and antisymmetrical modes in a free plate and to evaluate the Rayleigh and Stoneley equations.

We have calculated the dispersion of Rayleightype waves in plates for three solids which exhibit hexagonal symmetry: (a) beryl, (b) ice, and $(c)$ a laminated solid. These results, of course, apply also to any anisotropic solid having the same relationship between the elastic constants as one of the above examples.

Beryl was chosen in order to extend Stoneley's results to a free plate. Ice was chosen as an examplc of a solid which exhibits a rather strong anisotropy and because lake ice commonly forms with a vertical $c$-axis (the unique axis) orientation. The other axes are randomly oriented, but since they are all cquivalent the result is a large plate having the properties of a single crystal. Sea ice and some lake ice form with a $c$-axis horizontal orientation. Here again we have a large plate with transverse isotropy, but the effective elastic constants are not the same as for a single crystal. A finely laminated solid is transversely isotropic with the unique axis normal to the laminations; the effective elastic constants of a laminated solid must obey certain ordering rules which restricts the extent to which anisotropy may be approximated by layering. Table 1 gives the elastic constants used in computing these three cases.

To show more clcarly the effect of anisotropy we have also calculated the dispersion for equivalent isotropic ice, that is $\alpha=\alpha_{1}, \beta=\beta_{1}$, and for some intermediate cases.

For convenience we introduce the following 'anisotropy factors':

$$
\begin{array}{ll}
\varphi=c_{33} / c_{11} & \text { i.c. } \alpha_{2}{ }^{2}=\varphi \alpha_{1}{ }^{2} \\
\xi=\left(c_{11}-c_{12}\right) / 2 c_{14} & \text { i.e. } \beta_{1}{ }^{2}=\xi \beta_{2}{ }^{2} \\
\eta=\left(c_{11}-2 c_{44}\right) / c_{13} &
\end{array}
$$

Isotropic media have $\phi=\xi=\eta=1$.

Figure 1 shows the effect on the fundamental symmetric mode ${ }^{2}\left(M_{11}\right)$ of increasing all of the anisotropy factors from below 1 to the values attained by anisotropic ice. For this kind of anisotropy, i.e. $\varphi, \xi$, and $\eta$ increasing, the dispersion curve migrates uniformly toward higher phase velocity. Note the shift of the Airy phasc. The low-frequency limit of $M_{11}$ for an isotropic plate satisfics $c^{2} / \beta^{2}=4\left(1-\beta^{2} / \alpha^{2}\right)$. Therefore $\left(c_{n} / \beta\right) \leq 2$. For an anisotropic plate with the constants of ice, $(24)$ gives $\left(c_{p} / \beta_{1}\right)=2.045$. This agrees with the numerical evaluation of the general period equation.

Stoneley [1949] has previously noted that the Rayleigh velocity for an anisotropic solid can be higher than that for a Poisson solid. We note, in arldition, that it can be higher than is theoretically possible for an isotropic solid. This is true, in particular for solids with the constants of beryl and ice. Therefore, both ends of $M_{11}$ are greater than the theoretical maximum uncler the assumption of isotropy.

${ }^{2}$ We follow Tolstoy and Usdin's [195:3] modo designation convention. See also Ewing, Jardetzky, and Press [1!57, p. 283].

TABLE 1. Elastic and Anisotropic Parameters Adopted for Computation

\begin{tabular}{lllllcccl}
\hline Material & $c_{11} / c_{44}$ & $c_{33} / c_{44}$ & $c_{12} / c_{44}$ & $c_{13} / c_{44}$ & $\varphi$ & $\xi$ & $\eta$ & $\rho\left(\mathrm{g} / \mathrm{cm}^{3}\right)$ \\
\hline Beryl & 4.13 & 3.62 & 1.47 & 1.01 & 0.88 & 1.33 & 0.47 & 2.7 \\
Ice & 4.70 & 4.96 & 2.27 & 1.60 & 1.05 & 1.21 & 1.69 & 0.917 \\
Layered & 4.54 & 3.32 & 1.64 & 1.31 & 0.73 & 1.45 & 2.06 & 2.4 \\
\hline
\end{tabular}




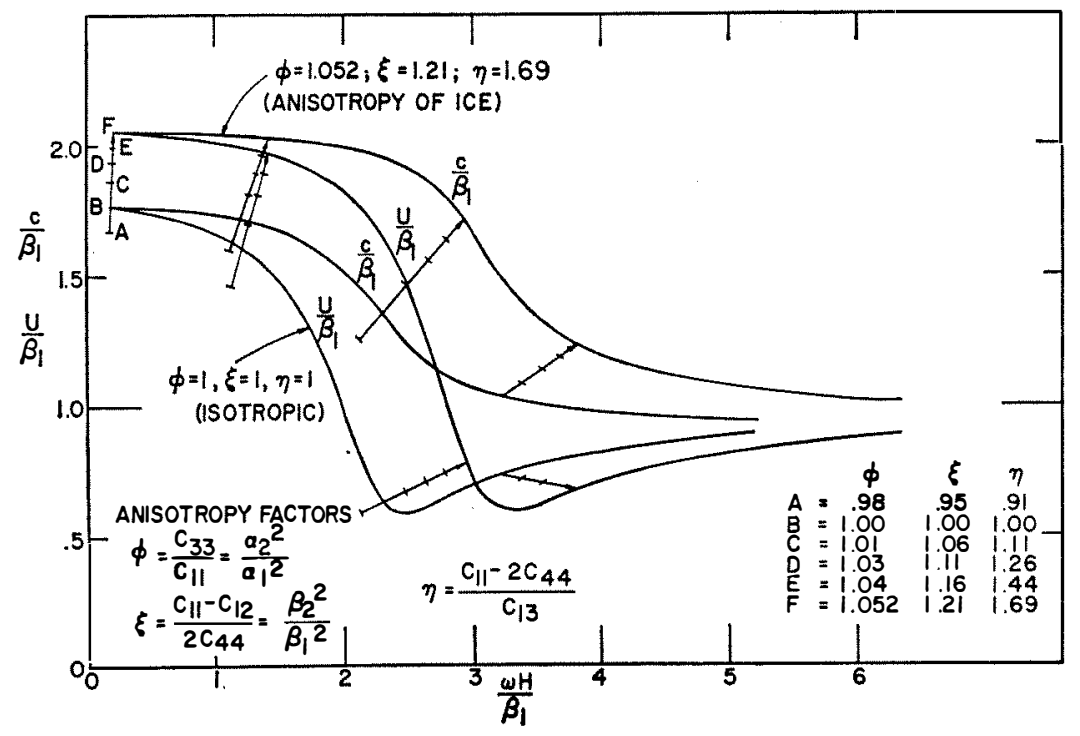

Fig. 1. Effect of anisotropy on $M_{11}$ in a transversely isotropic plate.

To pursue this point further we have plotted in Figure 2 the variation in the phase velocities of the four lowest modes of an isotropic plate as Poisson's ratio changes from 0.25 to 0.5 , the latter case indicating incompressibility. The data are from Satô [1951]. It is of interest to note that Poisson's ratio has a much greater effect on the symmetrical than on the antisymmetrical modes. This is to be expected since we have effectively held $\beta$ constant while changing $\alpha$, and the $M_{1}$ modes are chiefly compressional in nature while the $M_{2}$ modes tend to couple with an ideal free shear mode [Tolstoy, 1957]. Plotted for comparison are the four lowest modes for ice and $M_{11}$ for beryl. All the modes except $M_{21}$ (the flexural mode), which is not drawn, and the low-frequency end of $M_{11}$ for beryl are well outside the theoretical limit of isotropic plates. $M_{21}$ for ice deviates slightly from isotropic values at both ends.

In Figure 3 are shown the complete results for ice, for $M_{11}$ through $M_{22}$. Note the negative group velocity tail on $M_{12}$. This phenomenon has been discussed by Tolstoy [1957], who

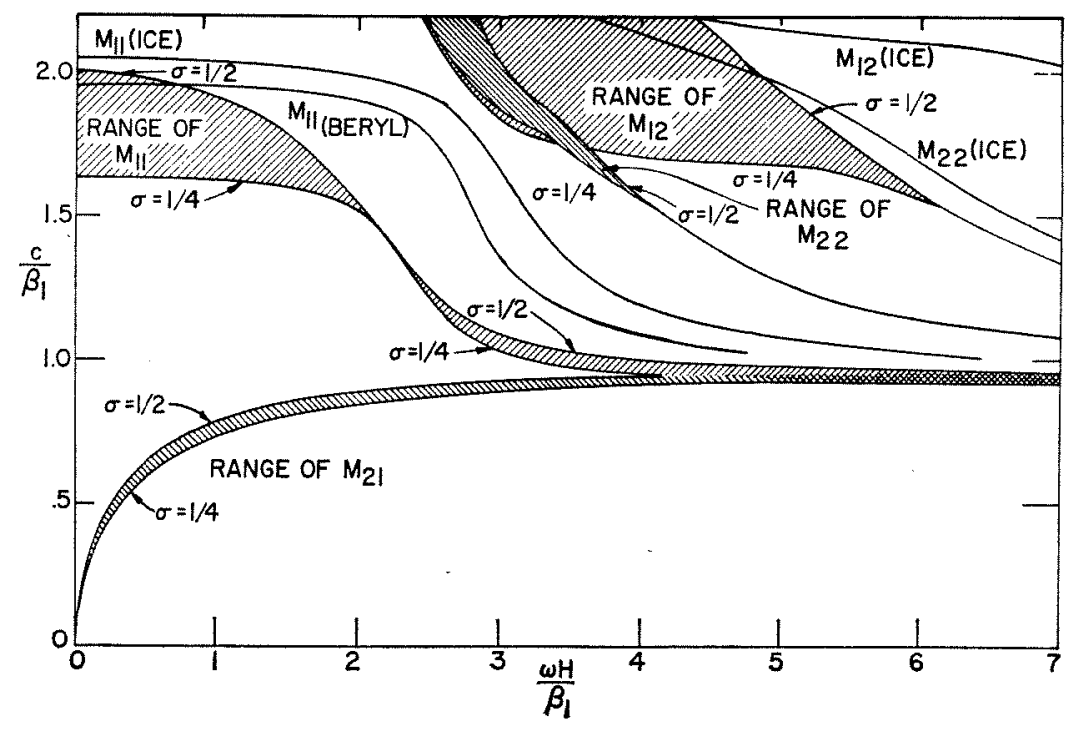

Fig. 2. Range of existence of $M_{11}$ through $M_{22}$ for $1 / 4<\sigma<1 / 2$ in isotropic plates and location of corresponding modes for anisotropic plates. 


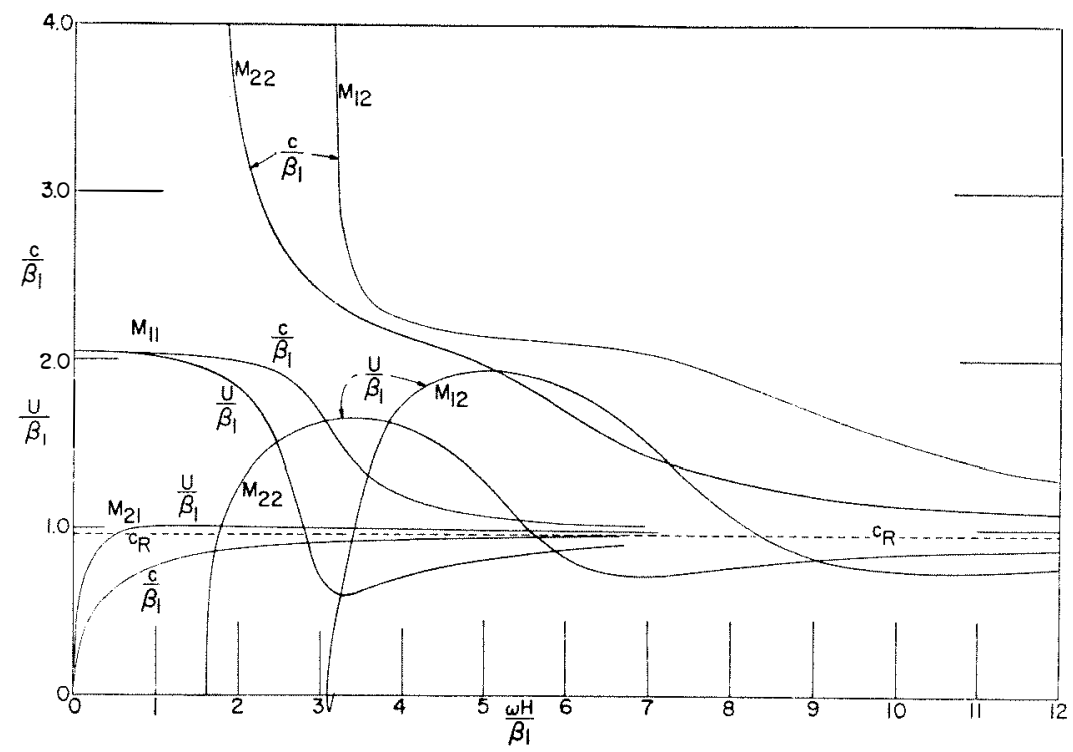

Fig. 3. Dispersion in a free anisotropic plate with the properties of ice.

attributes it to a negative phase velocity rather than to an actual backward propagation of energy.

Figure 4 gives the results for a laminated plate consisting of alternating layers of sandstone and limestone in the ratio 3 to 1 . The effective elastic constants for waves long compared with the lamination thickness in this medium have been computed by Postma [1955] and are listed in Table 1 . The anisotropy factors are $\varphi=0.733$, $\xi=1.450$, and $\eta=2.0576$, making the anisotropy of this material quite different from that of beryl or ice. Note the pulling down of the group velocity minimum and, in particular, the accentuated negative group velocity tail.

The Stoneley wave equation was evaluated numerically for $\rho_{2} / \rho_{1}=1.12$ (the water to ice density ratio) and several $\alpha^{*} / \beta_{1}$ ratios. As in the isotropic case, the Stoneley velocity is always a fraction of the slower of $\alpha^{*}, \beta_{1}$, but for the constants tested it is a larger fraction than for the corresponding isotropic case. For example, when $\alpha^{*} / \beta_{1}$ takes on the values 0.79 $0.9,1.0,1.1$, and 1.2 , the Stoneley velocity

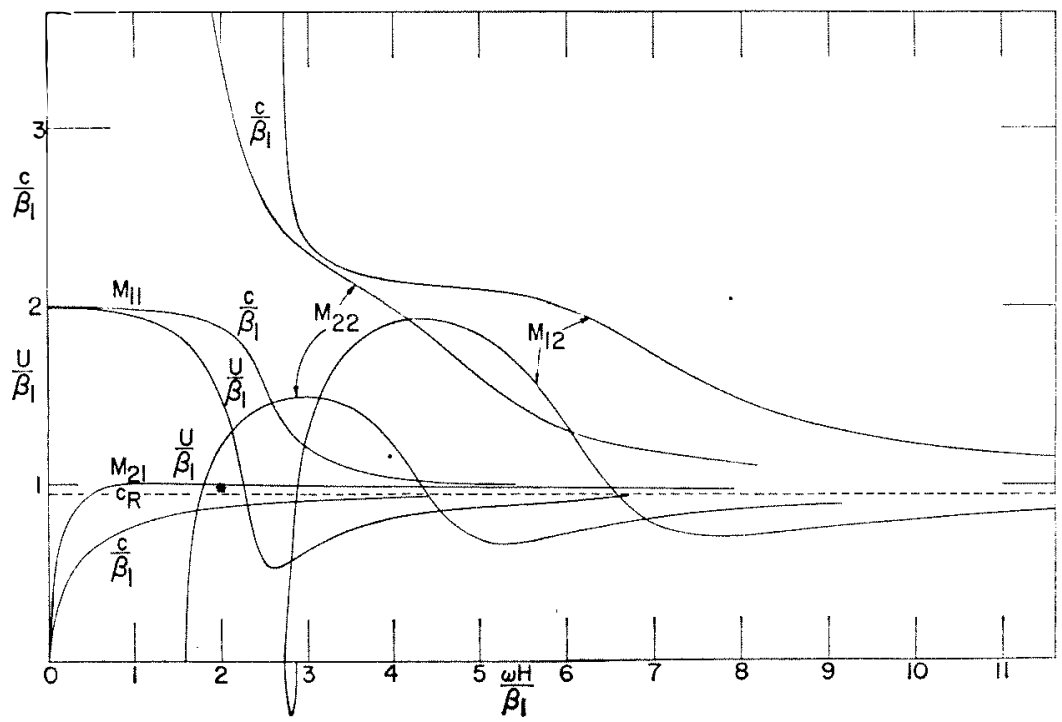

Fig. 4. 13ispersion in a laminated plate. 
$c_{s} / \beta_{1}$ is $0.70,0.74,0.76,0.78$, and 0.79 for the anisotropic case and $0.67,0.71,0.72,0.74$, and 0.75 for the isotropic case.

9. Matrix formulation of the general problem of a laycred medium. Following Haskell [1953] we now show how the solution of the wave propagation problem in a single anisotropic layer leads naturally to the $n$-layer problem. The normalized velocities and stresses in the $m$ th layer can be written (understanding an $e^{i(\omega t-k x)}$ factor):

$$
\begin{aligned}
& \dot{u} / c=\left[i k \operatorname{sh} \nu_{1_{m}} z U_{1_{m}}+i k c h \nu_{1_{m}} z U_{2 n}\right. \\
& \left.+i k \operatorname{sh} \nu_{2_{m}} U_{3_{m}}+i k c h \nu_{2_{m}} z U_{4_{m}}\right] \\
& \ddot{w} / \dot{e}=\left[-k \gamma_{1_{m}} c h \nu_{1_{m}} z U_{1_{m}}-k \gamma_{1_{m}} \operatorname{sh} \nu_{1, n} z U_{2_{m}}\right. \\
& -k \gamma_{2_{m}} c h \nu_{2_{m}} z U_{3_{m}}-k \gamma_{2_{m}} s h_{\nu_{2} z_{m}} z U_{4_{m}} \mathrm{~T} \\
& p_{z z}=i\left(\gamma_{1_{m}} \nu_{1_{m}} C_{m}-F_{m} k\right) s h \nu_{1_{m}} z U_{1_{m}} \\
& +i\left(C_{m}^{\gamma} \gamma_{1_{m}} \nu_{1_{m}}-I_{m} k\right) c l_{1 \nu_{1 m}} z\left(l_{2_{m}}\right. \\
& +i\left(\gamma_{2_{m}} \nu_{2 m} C_{m}-F_{m} k\right) s h \nu_{2_{m}} z U_{3_{m}}
\end{aligned}
$$

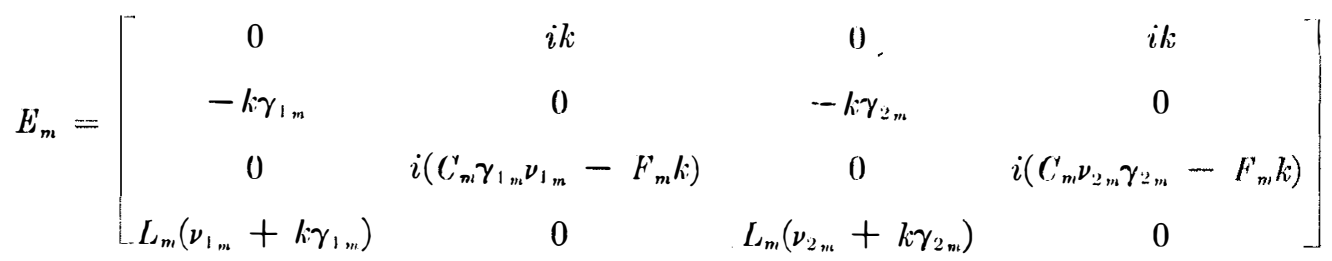

Setting $z=d_{m}$ we can write the relationship between the motion stress vector of the $m$ th interface and the $U_{i_{m}}$ :

$$
\left(\dot{u}_{m} / c, \dot{w}_{m} / r, p_{z: m}, p_{x i m}\right)=D_{m}\left(U_{m}, V_{m}, W_{m}, Z_{m}\right)
$$

Where $D_{m}$ is the matrix

$$
\begin{aligned}
& D_{m}=\left\{\begin{array}{cc}
i k s h \nu_{1_{m}} d_{m} & i k c h \nu_{1_{m}} d_{m} \\
-k \gamma_{1_{m}} c h \nu_{1_{m}} d_{m} & -k \gamma_{1_{m}} s h \nu_{1_{m}} d_{m} \\
\left\{i\left(\gamma_{1_{m}} \nu_{1_{m}} C_{m}-F_{m} k\right) s h \nu_{1_{m}} d_{m}\right\} & \left\{i\left(C_{m} \gamma_{1_{m} \nu_{1 m}}-F_{m} k\right) c h \nu_{1_{m}} d_{m}\right\} \\
L_{m}\left(\nu_{1_{m}}+k \gamma_{1_{m}}\right) c h \nu_{1_{m}} d_{m} & L_{m}\left(\nu_{1_{m}}+k \gamma_{1_{m}}\right) s h \nu_{1_{m}} d_{m}
\end{array}\right. \\
& i k s h \nu_{2 m} d_{m} \\
& -k \gamma_{2_{m}} c h \nu_{2_{m}} d_{m} \quad-k \gamma_{2_{m}} \operatorname{sh} \nu_{2_{m}} d_{m} \\
& \left\{i\left(\nu_{2_{m}} \gamma_{2_{m}} C_{m}-F_{m} k\right) s h \nu_{z_{m}} d_{m}\right\} \quad\left\{i\left(C_{m} \nu_{2_{m}} \gamma_{2_{m}}-F_{m} k\right) c h \nu_{2_{m}} d_{m}\right\} \\
& \left.L_{m}\left(\nu_{2_{m}}+\gamma_{2_{m}} k\right) c h \nu_{2_{m}} d_{m} \quad L_{m}\left(\nu_{2_{m}}+\gamma_{2_{m}} k\right) s h \nu_{2_{m}} d_{m}\right]
\end{aligned}
$$

where $C, L$, and $F$ are the elastic constants $c_{33}$, $\boldsymbol{c}_{14}$, and $\boldsymbol{c}_{13}$. The boundary conditions to be met at each solid-solid interface are that these four quantitics be continuous.

Taking $z=0$ at the interface $(m-1)$, we can write the linear relationship between the motion stress vector and the displacement where $\left(U_{1}, U_{2}, U_{3}, U_{4}\right)_{m}=\left(U_{m}, V_{m}, W_{m}, Z_{m}\right)$, and where $E_{m}$ is the matrix

$$
\begin{aligned}
p_{x z}=L_{m}\left(\nu_{1_{m}}+k \gamma_{1_{m}}\right) c h \nu_{1_{m}} z U_{i_{m}} \\
+L_{m}\left(\nu_{1_{m}}+k \gamma_{1_{m}}\right) \operatorname{sh} \nu_{1_{m}} z U_{2_{m}} \\
\left.+L_{m} \nu_{2_{m}}+\gamma_{2_{m}} k\right) c h \nu_{2_{m}} z U_{3_{m}} \\
\quad+L_{m}\left(\nu_{2_{m}}+\gamma_{2_{m}} k\right) \operatorname{sh} \nu_{2_{m}} z U_{4_{m}}
\end{aligned}
$$
cocfficients as

$$
\begin{aligned}
& \left(\dot{u}_{m-1} / c, \dot{w}_{m-1} / c, p_{z z m+1}, p_{x z m+1}\right) \\
& =E_{m}\left(U_{m}, V_{m,}, W_{m}, Z_{m}\right)
\end{aligned}
$$


The roefficionts $U$, may he oliminated between equations 433 and 46 , giving a lincar relationship) between the motion stress vector at the bottom and the top of the $m$ th layer:

$$
\begin{aligned}
& \left(\dot{u}_{m} / c, \dot{w}_{m} / c, p_{z z m}, p_{x z m}\right) \\
& \quad=D_{m} E_{m}^{-1}\left(\dot{u}_{m-1} / c, \dot{w}_{m-1} / c, p_{z z m-1}, p_{x z m-1}\right)
\end{aligned}
$$

Applying (47) recursively we may carry our solution down through a stack of $n$ layers. The development follows that of Haskell, and the properties of the matrices are the same as he discusses. By suitable alterations existing computcr programs for isotropic layers can be modified to handle anisotropic layers.

Calculations for the $n$-layer problem will be presented in future papers.

Acknowledgments. I wish to thank Dr. Frank Press for his advice and encouragement throughout this work, and to acknowledge helpful discussions with R. Phinney, C. Archambeau, and D. Harkrider.

This research was partially supported by contract 1)A-()4-495 Ord. 1808 (Model Seismology) of the U. S. Army Research Office, I urham, and contract Al-49(638)910 of the Air Force Technical Applications Center as part of the Advanced Research Projects Agency project Vela.

I gratefully acknowledge the support of a National Science Foundation predoctoral fellowship during the course of this work.

\section{REFERENCLS}

Ewing, W. M., W. S. Jardetzky, and F. Press,

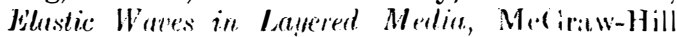
Borok Co., Now York, l!nst.
Haskell, N. A., Dispersion of surface waves on multilayered media, Bull. Scism. Suc. A m, 13, 17-331, 195:3.

Knopoff, L., On Rayleigh wave velocities, Bull. Seism. Soc. Am. 42, 307-308, 1952.

Love, A. E. H., A Treatise on the Mathematical. Theory of Elasticity, 4th ed., reprinted by Dover Publications, New York, 1944.

Mason, W. P., Physical Acoustics and the Properties of Solids, Bell Laboratory Series, D. Van Nostrand Co., Inc., Princeton, N. J., 1958.

Musgrave, M. J. P., The propagation of elastic waves in crystals and other anisotropic media, Repts. Progr. in Phys., 22, 74-96, 1959.

Phinney, R. A., Leaking modes in the crustal waveguide, 1, The oceanic $P L$ wave, J. Geophys. Research, 66, 1445-1469, 1961.

Postma, (i. W., Wave propagation in a stratified medium, Geophysics, 20, 780-806, 1955.

Press, F., and M. Ewing, Propagation of elastic waves in a floating ice sheet, T'rans. Am. Geophys. Union, 32, 673-678, 1951.

Satô, Y., Rayleigh waves projected along the plane surface of a horizontally isotropic and vertically aeolotropic elastic body, Bull. Earthquake Research Inst. ('T'okyo) 28, 23-30, 1950.

Satô, Y., Study on surface waves. II: Velocity of surface waves propagated upon elastic plites, Bull. Earthquake Research Inst. (T'okyo), 2:), 223-261, 1951.

Stonely, R., The seismological implications of :teolotropy in continental structure, Monthly Nolices Roy. Astron. Soc., Geophys. Suppl. 5, 222-232, 1949.

Tolstoy, I., and E. Usdin, Dispersive properties of stratified elastic and liquid media; a ray theory, Geophysics, 18, 844-870, 1953.

Tolstoy, I., Wave propagation in elastic plates; low and high mode dispersion, J. Acoust. Sor. A m., 苜!), $37.42,1957$.

(M:mmseript received Maty 25, 19661.) 